\title{
Virulence related sequences; insights provided by comparative genomics of Streptococcus uberis of differing virulence
}

\author{
Maqsud Hossain ${ }^{1}$, Sharon A Egan ${ }^{1}$, Tracey Coffey ${ }^{1}$, Philip N Ward ${ }^{2}$, Ray Wilson ${ }^{3}$, James A Leigh ${ }^{\text {* }}$ \\ and Richard D Emes ${ }^{1,4^{*}}$
}

\begin{abstract}
Background: Streptococcus uberis, a Gram-positive, catalase-negative member of the family Streptococcaceae is an important environmental pathogen responsible for a significant proportion of subclinical and clinical bovine intramammary infections. Currently, the genome of only a single reference strain (0140J) has been described. Here we present a comparative analysis of complete draft genome sequences of an additional twelve $S$. uberis strains.

Results: Pan and core genome analysis revealed the core genome common to all strains to be 1,550 genes in 1,509 orthologous clusters, complemented by 115-246 accessory genes present in one or more S. uberis strains but absent in the reference strain 0140J. Most of the previously predicted virulent genes were present in the core genome of all 13 strains but gene gain/loss was observed between the isolates in CDS associated with clustered regularly interspaced short palindromic repeats (CRISPRs), prophage and bacteriocin production. Experimental challenge experiments confirmed strain EF20 as non-virulent; only able to infect in a transient manner that did not result in clinical mastitis. Comparison of the genome sequence of EF20 with the validated virulent strain 0140J identified genes associated with virulence, however these did not relate clearly with clinical/non-clinical status of infection.

Conclusion: The gain/loss of mobile genetic elements such as CRISPRs and prophage are a potential driving force for evolutionary change. This first "whole-genome" comparison of strains isolated from clinical vs non-clinical intramammary infections including the type virulent vs non-virulent strains did not identify simple gene gain/loss rules that readily explain, or be confidently associated with, differences in virulence. This suggests that a more complex dynamic determines infection potential and clinical outcome not simply gene content.
\end{abstract}

Keywords: Mastitis, Streptococcus uberis, Comparative genomics, vru, de novo assembly, CRISPRs

\section{Background}

Implementation of the five point control measures for bovine mastitis including improved milking practice, post-milking teat disinfection, therapeutic and prophylactic antimicrobial administration, and the culling of persistently infected animals has made significant impact on the control of intramammary infections caused by contagious pathogens [1]. However, these measures are less effective in controlling infections from environmental pathogens, which continue to be a major hurdle in the control of

\footnotetext{
* Correspondence: james.leigh@nottingham.ac.uk; richard.emes@nottingham.ac.uk 'School of Veterinary Medicine and Science, University of Nottingham, Leicestershire, LE12 5RD Sutton Bonington, UK

${ }^{4}$ Advanced Data Analysis Centre, University of Nottingham, Nottingham, UK Full list of author information is available at the end of the article
}

mastitis. Streptococcus uberis, a Gram-positive, catalasenegative member of the family Streptococcaceae is an important environmental pathogen implicated in bovine mastitis, accounting for a significant proportion of subclinical and clinical intramammary infections [2]. Mastitis is defined as clinical when abnormality of the udder or secretion is observed, whereas, in subclinical mastitis the udder and the milk appears normal. The economic impact of both subclinical and clinical mastitis in the UK dairy industry is in excess of $£ 200$ million/ annum with worldwide economic loss estimated at US\$35 billion [3]. Control of S. uberis through vaccination based strategies therefore has the potential to dramatically improve both the economics of milk production and 
animal welfare [4]. Development of a vaccine against $S$. uberis has been hampered by a lack of information on the interaction between pathogen and the host [5]. This lack of knowledge is exemplified in the paucity of information on S. uberis strains at the genomic level. Whilst over 900 strains of $S$. uberis have been typed using multi locus sequence typing (MLST; http://pubmlst.org/suberis/), only a single genome sequence has been reported, from $S$. uberis, strain 0140J (accession number AM946015), selected as a typical virulent UK strain [6]. The genome of 0140J $(1,852,352 \mathrm{bp})$ is one of the smallest sequenced Streptococcus genomes which range from 1.8 Mb-2.3 Mb [6]. This suggests that through genome reduction, the 0140J genome has become condensed possibly reflecting restricted host-range. It is also possible that the 1,825 protein coding genes of $0140 \mathrm{~J}$ harbour potential virulence genes which are absent in non-virulent strains, or that loss of accessory genes present in other strains may be associated with the virulence of 0140J.

As an initial attempt to rectify this lack of genomic information and to identify the extent of genome variation between $S$. uberis strains, the genomes of an additional twelve strains were determined using high throughput sequencing approaches. The strains selected for sequencing are representative of the currently typed UK strains (Figure 1). Comparison of the predicted gene content was performed to identify the core genome shared by strains and the variable accessory genome between strains. Whilst the simplistic view that presence/absence of single genes or clusters of genes could be used to predict virulence or clinical status is tempting, our analysis suggest that this is not the case. In addition to bacterial factors, complexities such as bacterial population structure, host genetics and host immune status are likely to play a role in the linking of clinical status and bacterial virulence.

\section{Methods}

\section{DNA isolation and genome sequencing}

Bacteria from a range of clinical and sub-clinical isolates (see Additional file 1) were inoculated into Todd-Hewitt Broth and grown at $37^{\circ} \mathrm{C}$ overnight with DNA extracted from cultures as previously described [7].

\section{Genome assembly and annotation}

Library preparation and sequencing of each strain was conducted at DeepSeq, Queens Medical Centre, Nottingham, UK. $1 \mu \mathrm{g}$ of high molecular weight $S$. uberis genomic DNA were used to prepare Illumina libraries using the TrueSeq DNA LT Sample Prep Kit (Cat. no, FC-121-2001) as described in TrueSeq DNA sample preparation guide with the following modifications. DNA fragmentation was carried out in covaris S2 using the following parameters: Duty cycle - 10\%, Intensity - 5, cycles per burst - 200, Time -45 seconds, Mode - Frequency sweeping and temperature $-6^{\circ} \mathrm{C}$. Gel method was used to size-select adapter ligated DNA to 600-700 bp to generate libraries with insert length of 500-600 bp for the increased MiSeq read length. Sequencing was performed on the MiSeq platform with V2 chemistry (Cat. no, MS-102-2003) to generate

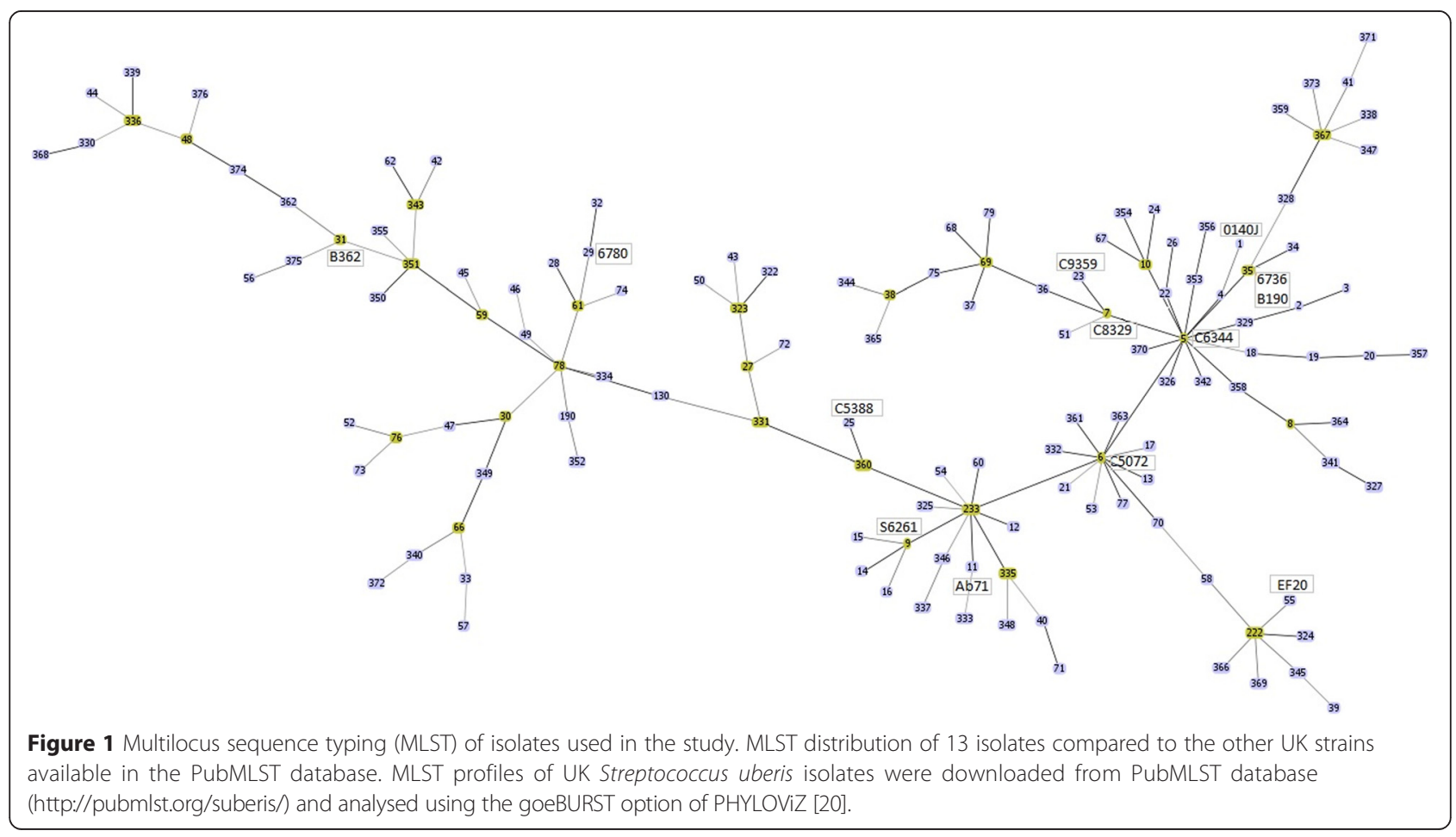


$2 \times 250$ bp paired end reads. The average number of reads per strain was 902,651. Reads were used to generate assemblies using Velvet (version 1.2.10) [8]. Maximum N50 was used as the measure to determine optimal K-mer length using Velvetoptimiser (https://github.com/ Victorian-Bioinformatics-Consortium/VelvetOptimiser) a minimum coverage of $10 \mathrm{x}$ was selected and the -exp_cov option 'auto' was used. CONTIGuator [9] was used to map resulting contigs to the reference genome 0140J for comparative analysis of genomic regions.

Assembled contigs were annotated using the Rapid Annotations using Subsystem Technology (RAST) server [10]. The pan-genome analysis pipeline (PGAP version 1.02) [11] was used for identification of orthologous genes between the twelve sequenced genomes and the reference genome 0140J using the Gene Family (GF) method (50\% coverage and with an e-value cut-off $1 \mathrm{e}-10)$. Similarity of the samples based in gene presence/absence was conducted using hierarchical clustering. The pvclust package (http://cran.r-project.org/web/packages/pvclust/) was using a correlation distance measure and average agglomeration method.

PILER-CR [12] and CRISPRs web server [13-15] was used for rapid identification and classification of clustered regularly interspaced short palindromic Repeats (CRISPRs). The phage search tool (PHAST) [16] was used to identify, annotate and graphically display prophage sequences within the draft genomes. MUSCLE [17] was used for multiple alignments. The webserver snpTree [18] was used to identify SNP positions trees from the concatenated 1,377 core genes of 13 isolates. PhyML [19] was used for the generation of phylogenetic trees using a GTR model estimated gamma distribution and 4 substitution rate categories. 200 bootstrap replicates were conducted.

Multilocus Sequence Typing (MLST) profiles of UK Streptococcus uberis isolates were downloaded from PubMLST database (http://pubmlst.org/suberis/) and analysed using the goeBURST option of PHYLOViZ [20]. Assembled contigs are available at GenBank under the accession numbers provided in Table 1. RAST annotation are available as Additional file 2.

To determine the stability of pan/core genomes, the pan genome (total number of genes identified within a group of samples) and core genome size (those genes shared by a group of samples) was determined when between 2 and 13 genomes were combined in random order. For each combination size ( $2 \ldots 13$ genomes) 1000 permutations were conducted.

\section{Challenge of lactating dairy cattle with S. uberis 0140J or EF20}

To ascertain the virulence of two S. uberis strains, 5 dairy cows aged between 24-30 months were selected for experimental challenge at 4-8 weeks post calving, using a well-established intramammary infection model. Criteria for selection were: absence of signs of mastitis, no history of mastitis during the current lactation and absence of bacteria in milk samples taken $24-48 \mathrm{~h}$ prior to challenge with the associated somatic cell count (SCC) below 100,000 cells $/ \mathrm{ml}$. Animals were challenged in two mammary quarters after morning milking by infusion of $1 \mathrm{ml}$ of pyrogen-free saline (Sigma) containing approximately $1 \times 10^{3} \mathrm{CFU}$ of $S$. uberis 0140J or EF20 prepared as previously described [7].

Following challenge, animals were milked and inspected twice daily. Milk and udder quarters were assessed to determine the severity of disease using predetermined criteria for clinical end points (clotted and discoloured milk and/or udder quarter swollen or causing discomfort on palpation) as previously described [7]. Milk collected from challenged quarters at each milking (up to $48 \mathrm{~h}$ ) post-challenge was assessed for bacterial numbers and somatic cell counts. The number of viable bacteria present was estimated by plating of each milk sample onto ABA

Table 1 De novo assembly statistics of 12 Streptococcus uberis isolates

\begin{tabular}{|c|c|c|c|c|c|c|}
\hline Isolate & GenBank accession No. & Sequence size & Number of contigs (>200 bp) & Shortest contig size & Longest contig size & n50 \\
\hline EF20 & JANW00000000 & 1933244 & 18 & 369 & 1013731 & 1013547 \\
\hline 6736 & JATB00000000 & 1893525 & 28 & 392 & 424329 & 386092 \\
\hline 6780 & JATD00000000 & 1960858 & 26 & 409 & 404720 & 340682 \\
\hline Ab71 & JATK00000000 & 1849250 & 12 & 449 & 792968 & 425420 \\
\hline B190 & JATE00000000 & 1881868 & 20 & 450 & 1043356 & 1043142 \\
\hline B362 & JATC00000000 & 1912672 & 31 & 429 & 995597 & 995383 \\
\hline C5072 & JATI00000000 & 1906212 & 19 & 431 & 1021112 & 1020928 \\
\hline C5388 & JATF00000000 & 1837997 & 13 & 409 & 1030559 & 1030365 \\
\hline C6344 & JATA00000000 & 1907431 & 15 & 431 & 999672 & 999478 \\
\hline C8329 & JATG00000000 & 1923969 & 20 & 389 & 830811 & 455116 \\
\hline C9359 & JATJ00000000 & 1837707 & 11 & 450 & 1030658 & 1030464 \\
\hline S6261 & JATH00000000 & 1868255 & 26 & 429 & 613122 & 392636 \\
\hline
\end{tabular}


and the number of somatic cells present in milk samples was determined using a DeLaval portable cell counter in line with the manufacturer's instructions.

\section{Results and discussion}

General features of Streptococcus uberis genomes

Of the 12 strains sequenced, seven were classed as clinical and five sub-clinical isolates based on the status of dairy cow health during strain collection (Additional file 1). The combined length of the assembled contigs range from $1,837,707$ bp to $1,960,858$ bp (Table 1 ). This range spans the $1,852,352 \mathrm{bp}$ of the 0140J reference strain and falls within the $1.8 \mathrm{Mb}-2.3 \mathrm{Mb}$ predicted by Ward et al [6]. The median size of the genomes from each group differ (clinical median length $1,887,233.5 \mathrm{bp}$, subclinical median length $1,903,098.5 \mathrm{bp}$ ). However, the variation within both groups is large (interquartile range clinical: $74,423.5 \mathrm{bp}$, subclinical: $55,387.5 \mathrm{bp}$ ) and as a result there is no significant difference of total genome size (as measured by total assembled contig size) between the two groups. Therefore this does not support the assuption that clinical strains exhibit a reduced size genome like 0140J. This may reflect the relatively loose definition of clinical vs subclinical strains. To investigate the relationship of genome type to virulence phenotype, the representative avirulent strain EF20 was compared in an experimental infection model, to the clinical virulent 0140J strain. All challenged quarters became infected and shed bacteria at detectable levels from the first milking (12 hours post challenge). Those challenged with strain 0140J shed bacteria at $10^{4} \mathrm{cfu} / \mathrm{ml}$ of milk $12 \mathrm{~h}$ post-challenge) rising to $10^{6}-10^{7} \mathrm{cfu} / \mathrm{ml}$ of milk by $48 \mathrm{~h}$ post challenge (Figure $2 \mathrm{a}$ ). In contrast, those challenged with strain EF20 shed considerably fewer bacteria $\leq 10^{3} \mathrm{cfu} / \mathrm{ml}$ and typically declining to around $10^{2} \mathrm{cfu} / \mathrm{ml}$ of milk by $48 \mathrm{~h}$ post challenge. The speed of cellular infiltration into the mammary gland in response to infection with either strain was similar, however the magnitude of the infiltration was 10 fold less following challenged with strain EF20. The somatic cell count detected following challenge with strain 0140J was similar to those reported previously for this strain $[7,21,22]$ (Figure 2b). The levels of cellular infiltration and bacterial colonisation for each challenged quarter showed a significant positive correlation $\left(R^{2}=0.404, P<0.001\right)$ over the time-course of the experiment. Acute clinical signs of mastitis (change in milk composition, swollen and inflamed udder quarter) occurred in all animals challenged with strain 0140J (Figure 2c). In contrast, those challenged with strain EF20 showed few, if any, changes in milk composition and/or quarter appearance, thus substantiating and adding detail to the previous data [23] regarding the nature of virulence of these two strains and confirming their suitability for direct comparison at the genomic level in the elucidation of virulence related features.
The draft genome of EF20 consists of a slightly larger genome compared with 0140J. The 1,933,244 bp assembled draft genome has a $\mathrm{G}+\mathrm{C}$ content of $36.5 \%$ comparable to the $36.6 \% \mathrm{G}+\mathrm{C}$ content in 0140J. In line with its larger genome, EF20 has an increased number of predicted coding sequences of 1,957 compared to the 1,825 of 0140 J. At the gross level, comparative analysis of EF20 and 0140J revealed high-level synteny disrupted by a large number of gene gain/loss and recombination events (Figure 3). The larger predicted gene count in the EF20 genome is not simply addition within EF20 and/or loss in 0140J. Whilst 1,629 annotated genes are common between the two strains, strain specific genes are present in both 0140J (145 genes present in 0140J not in EF20) and EF20 (222 genes present in EF20 not in 0140J) (Additional file 3:orthologous clustering). The copper metabolism operon SUB1180SUB1184 was absent in EF20, however, growth comparison studies in restrictive levels of copper does not appear to differentially affect the rate of growth of either strain (results not shown). This may be due to a compensatory gene duplication event of the copper operon located in the region SUB1462-SUB1464. Following sequencing of the 0140J strain, a list of suggested virulence genes were proposed for S. uberis [6]. Many of these are found in the EF20 genome and appeared to be intact and hence presumably functional, including SUB1111 (Fibronectin- binding protein), SUB1273 (Hemolysin like protein), SUB1154 (C5a peptidase precursor), SUB0881 (Sortase A), SUB0145 (Lactoferrin binding protein), SUB1095 (Collagen like surface-anchored protein), SUB1635 (SUAM protein), SUB1785 (PauA Streptokinase precursor) suggesting that the simple presence of these genes is insufficient to explain virulence in $S$. uberis. Comparison of the inferred differences between metabolic subsystems highlights multiple differences (Additional file 4: Subsystem enrichment). Tests for association $\left(\chi^{2}\right.$ with Benjamini-Hochberg multiple hypothesis correction $\mathrm{p} \leq 0.05)$ identifies ten subsystems enriched in either 0140J or EF20. Bacterial checkpoint-controlrelated cluster, F0F1-type ATP synthase, Fructose and Mannose Inducible PTS, Restriction-Modification System, Formaldehyde assimilation: Ribulose monophosphate pathway and Lysine Biosynthesis DAP Pathway subsystems are over-enriched in 0140J compared to EF20. Whereas, D-Tagatose and Galactitol Utilization, Phage replication and Cadmium resistance subsystems are enriched in EF20 compared to 0140J.

To further elucidate the complexity of strain differences in S. uberis, the genome content of an additional 12 sequenced strains were compared. The sequencing of multiple strains of a given bacteria allows the definition of the core genome, the total number of genes shared by all strains sequenced, this was found to be 1,550 genes between the 12 assembled strains and 0140J 

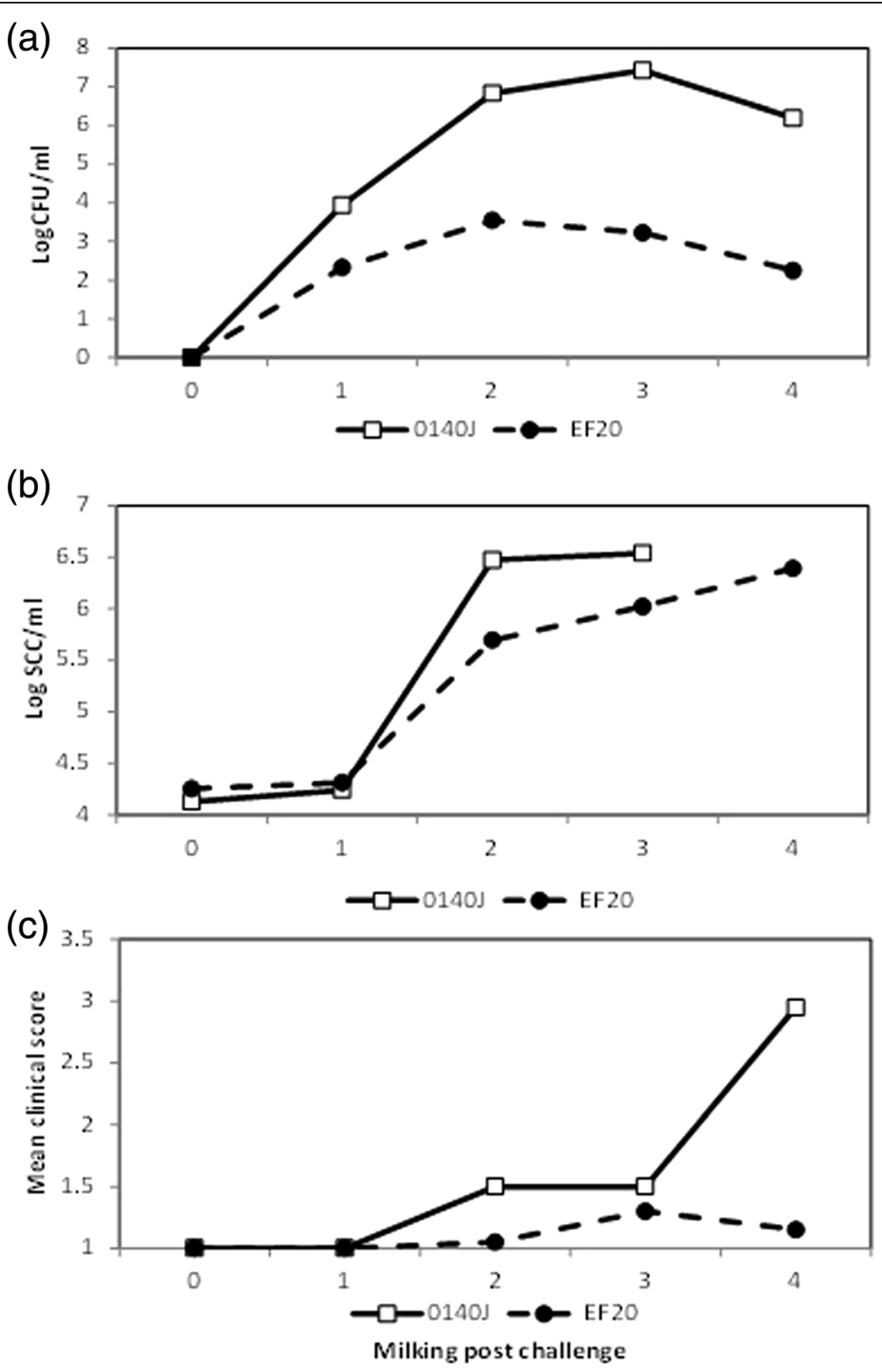

Figure 2 Bacterial isolation, somatic cell count and clinical response following challenge with S. uberis 0140J and EF20 in dairy cattle. Geometric mean value obtained after challenge of animals with either S. uberis strain 0140J $(n=10)$ or the EF20 $(n=10)$. (a) Bacterial recovery of S. uberis at each milking following challenge, measured by cfu/ml of milk (b) Cellular influx at each milking following $\mathrm{S}$. uberis challenge, measured by somatic cell count/ml of milk (c) Combined clinical scores from clinical manifestations following S. uberis challenge. Data is represented as the arithmetic mean of clinical scores given for the appearance of the quarter and appearance of the milk as previously described [7].

(Additional file 3:core_genome) identified in 1,509 orthologous clusters (Additional file 3: RAST_orthologous_ clusters). By repeatedly comparing the shared core genome clusters obtained from 1000 random combinations of strains we can see that with two genomes the median shared core genome size is 1,635 genes with a variance (inter-quartile range) of 44 genes. By comparison of 5 assembled genomes the median is 1,560 with inter-quartile range of 29 genes. If 10 genomes are compared the core genome size plateaus at 1,521 genes and the interquartile range is 16 genes (Figure 4a). The identified $S$. uberis core genome contained gene clusters or operons of genes essential for cellular growth and replication including those involved in cell wall and capsule synthesis, cell division, cell cycle regulation and cell signalling, membrane transport (protein secretion systems), RNA/DNA metabolism, metabolism of cofactors, aromatic compounds, amino acids and derivatives (arginine, lysine, threonine metabolism), phosphorus, fatty acid and lipids and carbohydrate uptake and utilization. 


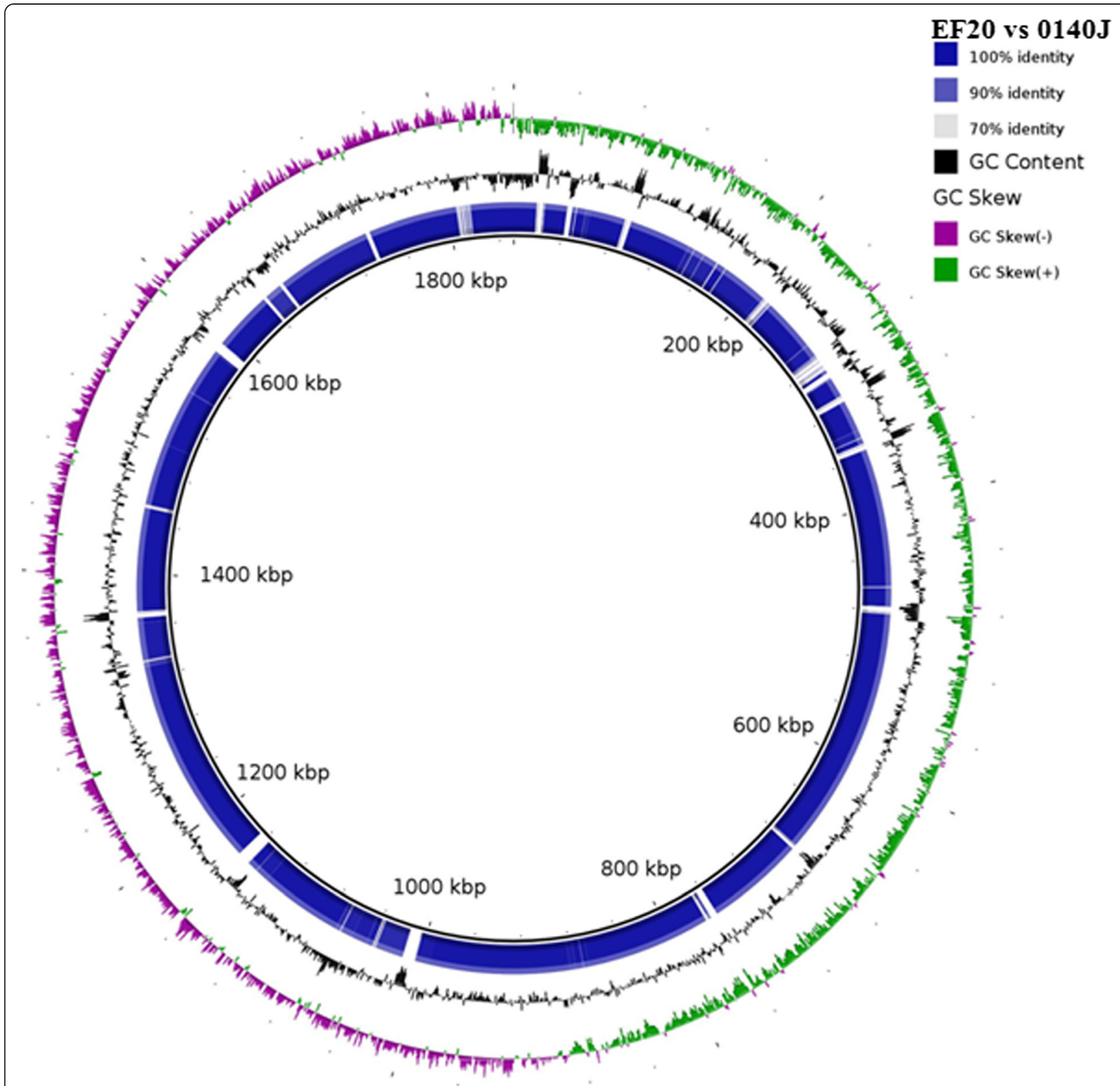

Figure 3 Sequence similarity comparison of 0140J and EF20. The innermost dark circle represents the reference genome 0140J (genome size $1852,352 \mathrm{bp}$ ) and the blue circle surrounding the reference genome represents the genome of EF20. Image generated using BRIG [40].

\section{Analysis of the pan genome}

The pan genome size (total number of genes within a group of genomes) was determined to give a measure of the relative complexity within the $S$. uberis genomes. With 10-12 S. uberis genomes the number of novel genes identified with the addition of an additional genome slows but does not plateau (Figure 4b), suggesting an open pangenome [24]. Together these comparisons of the core and pan genomes suggest that sequencing the relatively small number of strains has captured the majority, but not all of variation of $S$. uberis genomes.
Direct comparison of all newly sequenced genomes with the available 0140J strain identifies between 115-246 accessory genes present in one or more $S$. uberis strains but not present in 0140J. Following the assumption that the genomes of clinical strains are smaller, the clinical isolates were found to have slightly fewer accessory genes (median 180.5) than the sub-clinical isolates (median 193.5), however again these differences were not statistically significant. In addition, hierarchical clustering of the strains based on their shared accessory genome content, does not group strains by clinical status (Figure 5). 

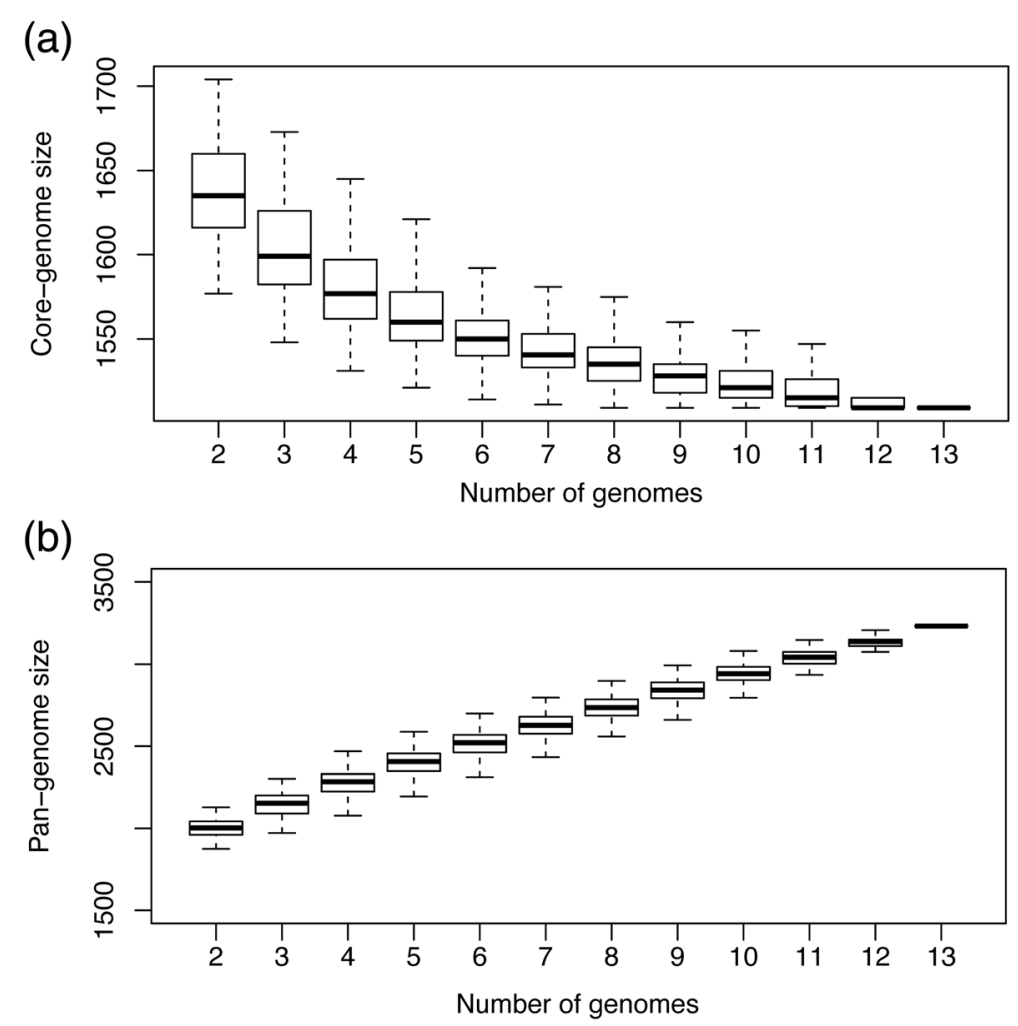

Figure 4 Relationship between gene count and genome size. To determine the stability of pan/core genomes, (a) the core genome size (number of common genes within samples) and (b) pan genome size (total number of different genes identified within samples) was determined when between 2 and 13 genomes were combined in random order. For each combination size (2..13 genomes) 1000 permutations were conducted. The boxplots represent the median and interquartile ranges of 1000 permutations, whiskers extend to the value extremes.

Alignment of 1,377 concatenated core genes comprising $1,294,803$ nucleotides identified 12,982 variable sites (SNPs). To account for possible recombination affecting the phylogeny the Phi-test [25] was conducted on 500 base windows of the core genome. Following a Bonferroni correction, those windows with significant evidence $(p<0.05)$ of recombination were masked from the alignment (1386 windows total 6,930,000 bases masked) and the phylogeny determined using PhyML as described in the methods. Whilst the bootstrap support was affected, the topology of the tree was unaffected by the masking of possible recombination regions. In agreement with the

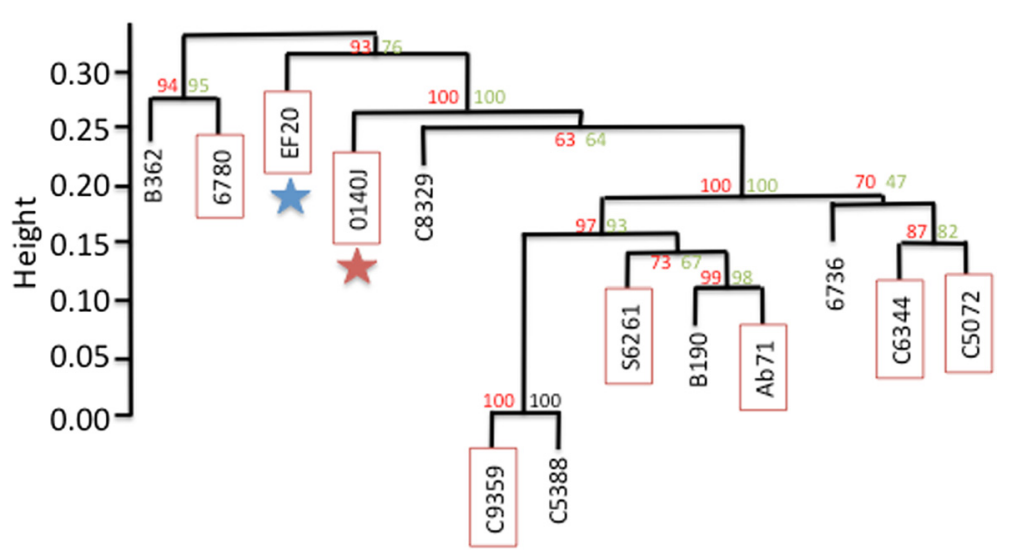

Figure 5 Pan genome clustering of $S$ uberis isolates. Similarity of the samples based in gene presence/absence was conducted using hierarchical clustering (pvclust) (http://cran.r-project.org/web/packages/pvclust/). The red star denotes the virulent strain 0140J, blue star, non-virulent strain EF20. Strains initially recorded as clinical isolates are boxed in red. 
shared gene content, clustering based on the presence or absence of shared SNPs discriminates the genomes based on ST clonal complex rather than by clinical status (Figure 6).

\section{Comparison of virulence factors}

As with the previous comparison of EF20 and 0140J the reported virulence genes SUB1111, SUB1273, SUB1154, SUB0881, SUB0145, SUB1095, SUB1635 and SUB1785 were found in the core genome of all other sequenced strains. Whilst the presence of these genes is conserved in clinical and non-clinical strains, they do exhibit a range of sequence conservation between strains. PauA (SUB1785) and Hemolysin-like protein (SUB1273) are very well conserved between the 12 strains with only 3 and 5 variable sites respectively in genes of over $800 \mathrm{bp}$ (Table 2a). In contrast, Collagen like surface-anchored protein (SUB1095) and Lactoferrin binding protein (SUB0145) are highly variable with over 0.4 SNPs/bp. The reference strain 0140J contains a single copy of gene (SUB0881) homologous to sortase A (srtA). Mutation of the srtA a transamidase which covalently anchors a subset of proteins to peptidoglycan on the surface of S. uberis has been shown to reduce S. uberis infective potential [7]. This suggests that the sortase-anchored proteins contain one or more virulence factors important for establishment of infection. The sortase-anchored proteins of S. uberis are known to contain a conserved amino acid LPxxG or LPxxxD motif [7]. Using the presence of either of these motifs together with a predicted secretory leader motif, 10 genes (SUB0135, SUB0145, SUB0207, SUB0241, SUB0826, SUB0888, SUB1095, SUB1154, SUB1370 and SUB1730) were identified as predicted potential sortase anchored proteins. Nine of these have been confirmed by comparative proteomic analysis [7], whilst SUB0241 was not. Sortase anchored genes SUB0145, SUB1095 and SUB1154 have been shown to be important in infection and have been proposed to be virulence candidates [7]. $S$. uberis mutants in which these genes were inactivated were attenuated in their ability to infect cattle [7]. Of these, SUB0145 and SUB1095 are highly variable between strains (Table 2b) suggesting that variation between them is

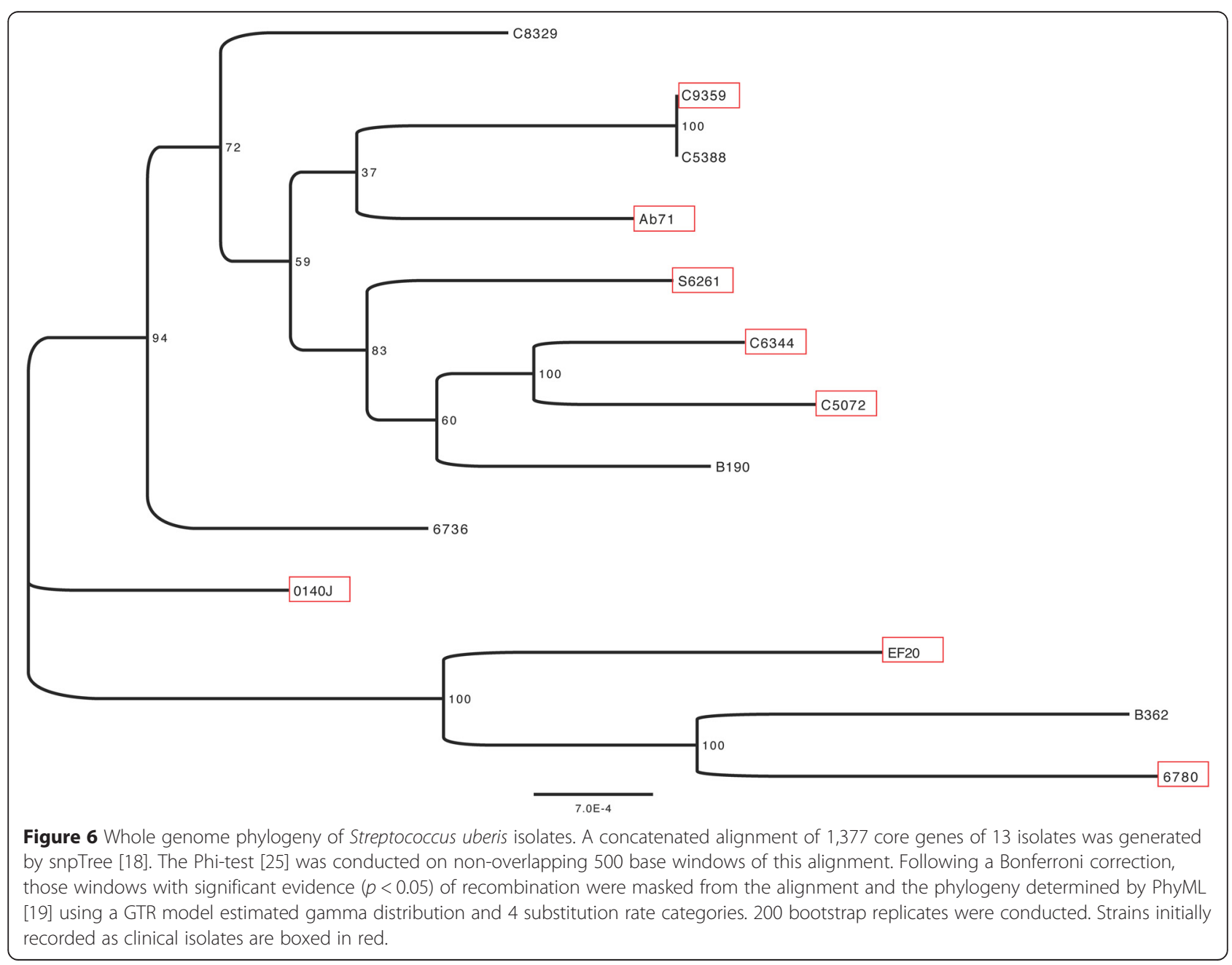


Table 2 SNP distribution of (a) known virulence genes (b) sortase anchored proteins

\begin{tabular}{|c|c|c|c|c|c|}
\hline Gene & Gene function & SNP count & Gene size (bp) & Alignment size (bp) & SNP/bp \\
\hline \multicolumn{6}{|l|}{ (a) } \\
\hline SUB1785 & PauA (Streptokinase precursor) & 3 & 861 & 861 & 0.003 \\
\hline SUB1273 & Hemolysin like protein & 5 & 828 & 828 & 0.006 \\
\hline SUB0881 & Sortase A (srtA) & 8 & 759 & 759 & 0.011 \\
\hline SUB1154 & C5a peptidase precursor & 73 & 3480 & 3362 & 0.022 \\
\hline SUB1635 & SUAM protein & 52 & 2637 & 2637 & 0.020 \\
\hline SUB1111 & Fibronectin- binding protein & 42 & 1653 & 1653 & 0.025 \\
\hline \multicolumn{6}{|l|}{ (b) } \\
\hline SUB0135 & putative fructan beta-fructosidase precursor & 36 & 3810 & 3810 & 0.009 \\
\hline SUB0145* & lactoferrin binding protein & 552 & 1819 & 1352 & 0.408 \\
\hline SUB0207 & putative surface-anchored protein & 28 & 1500 & 1500 & 0.019 \\
\hline SUB0241 & putative surface-anchored 2',3'-cyclic-nucleotide 2'-Phosphodiesterase & 48 & 2478 & 2457 & 0.020 \\
\hline SUB0826 & putative surface-anchored subtilase family protein & 208 & 4492 & 4418 & 0.047 \\
\hline SUB0888 & putative surface-anchored protein & 20 & 852 & 837 & 0.024 \\
\hline SUB1095* & collagen-like surface-anchored protein & 375 & 1452 & 935 & 0.260 \\
\hline SUB1154 & C5A peptidase precursor & 73 & 3480 & 3435 & 0.021 \\
\hline SUB1370 & putative zinc carboxypeptidase & 58 & 3225 & 3213 & 0.018 \\
\hline SUB1730 & putative surface-anchored protein & 128 & 1191 & 1020 & 0.125 \\
\hline
\end{tabular}

*putative virulence gene and sortase substrate.

maintained by natural selection in turn suggesting that this may be driven by interaction with the host immune system. Conversely, SUB1154 is more conserved between strains (0.021 SNPs/bp aligned). Using RAST to transfer annotation from the 0140J reference suggested that SUB1095 was unique to 0140J. Since this is an important virulence factor we manually checked whether this gene is truly absent from all other strains. However, using the Rapid Annotation Transfer Tool (RATT) a SUB1095 ortholog could be detected in all genomes sequenced suggesting that RAST was unable to annotate this gene due to the highly variable nature of SUB1095.

\section{Analysis of the has operon}

The hyaluronic acid capsule of S. pyogenes has been found to play a significant role in the pathogenesis of invasive Group A Streptococcus (GAS) bacteria [26], [27]. S. uberis strains isolated from cases of bovine mastitis display variable amounts of hyaluronic acid capsule [6] suggesting that the capsule may be associated with infection. However, Field et al 2003 showed that capsule negative mutants can still cause mastitis [21] and the availability of more capsule in clinical isolates than the environmental isolates [28] may be due to the fact that capsule prevents desiccation in the environment and allows it to persist longer, increasing chances of subsequent infection or even gut colonisation. In S. uberis 0140J the arrangement of the hyaluronic acid biosynthetic genes comprising the has operon, differs from the typical
"hasABC" arrangement common to GAS [28]. HasA (SUB1697) encoding hyaluronan synthase and hasB (SUB1696) encoding UDP-glucose dehydrogenase are arranged as in other GAS. However the has $C$ homologue (SUB1691), encoding UDP-glucose pyrophosphorylase, is encoded in the reverse orientation and separated from has $\mathrm{AB}$ by approximately $3 \mathrm{~kb}$ of genome encoding CDSs thought to be unrelated to capsule biosynthesis [6]. It is unlikely that this arrangement affects capsule production, as in GAS capsule is dependent only upon functional has $A$ and hasB, but not hasC [29]. All the isolates sequenced here except strain B362 have has $\mathrm{ABC}$ in a similar arrangement to that found in 0140J. In nine $S$. uberis strains a paralog of hasB (SUB1027) was identified. The non-capsular, non-virulent isolate EF20 lacks SUB1027 and this gene is also missing from isolates B362, 6780 and B190.

S. uberis SUB0144 is a homologue of the S. pyogenes virulence regulatory gene mga. SUB0144 ( $\mathrm{rru}$ ) of S. uberis has been found to regulate a number of virulent genes including hasA and hasB1 (SUB1696 and SUB1697), Lbp (SUB0145), SclB (SUB1095) and PauA (SUB1785) and inactivation of $v r u$ resulted in reduced ability to colonize the mammary gland as well as reduced clinical signs of mastitis compared with the wild-type strain [30]. Moreover, Flores et al have shown that a 12-bp deletion in the VNTR region of $m g a$ promoter at positions -63 to -75 alters GAS virulence, resulting in asymptomatic carrier phenotype [31]. In $S$. uberis we observe a deletion of 
five bp in a similar region of vru (position -75 to -79). This deletion was seen in several isolates including the nonvirulent strain EF20 and 6736. A four bp deletion was seen in positions -76 to -79 in five isolates Ab71, C9359, B362, C5388 and C8329 (Figure 7). Whilst not perfectly segregating, this deletion was found in most of the sub-clinical isolates and only in three clinical isolates. Hence, variation in this region may play an important role in the regulation of this regulatory gene and in turn influence the host-pathogen interaction.

\section{Analysis of CRISPR-Cas proteins}

The CRISPR-Cas (clustered regularly inter- spaced short palindromic repeats-CRISPR- associated proteins) identified in approximately $40 \%$ bacteria and most archaea, are genomic regions involved in adaptive immunity against invading genetic elements such as viruses [32-37]. CRISPER-Cas genes were detected in all strains except EF20 and 0140J. In strains other than EF20 and 0140J, The type II system [38] which includes the 'HNH'-type (Streptococcus-like) comprising Cas9/Csn1 (a single, large protein) was located in a conserved region between genes SUB1084-SUB1085 (Figure 8). An additional Type III cas1-cas6 gene set [38] was found in the isolate B362 inserted between genes SUB0330-SUB0333. In isolates EF20, Ab71 and C5072 an insertion event of two genes, homologous to Streptococcus pneumoniae integrative and conjugative elements (ICE) are present in the homologous region between SUB1084-SUB1085 (Figure 8).

\section{Analysis of prophage regions}

Growing evidence suggests the significant role of prophage regions in the virulence and evolution of many bacteria. For example, lysogeny has been found to contribute to the virulence of a number of organisms including Vibrio cholerae, Salmonella enterica, Escherchia coli, Clostridium botulinum, Corynebacterium diphtheriae, Staphylococcus aureus and Streptococcus pyogenes [39]. Analysis of prophage regions shows that among the thirteen isolates seven had intact prophage regions (Table 3). The nonvirulent isolate EF20 and subclinical isolate B362 did not have any identified prophage regions. An incomplete prophage region is evident in the region SUB1818-SUB1840 of the isolate $0140 \mathrm{~J}$ and this region is variable in most of the isolates. The diversity of these prophage regions may contribute to the adaptation of lysogens to new hosts.

\section{Analysis of bacteriocin production}

Bacteriocins are proteinaceous antibiotics produced by bacteria, which kill or inhibit the growth of other bacteria, often providing an advantage in competitive colonization environments. Uberolysin is a novel cyclic bacteriocin produced by $S$. uberis encoded by the operon spanning SUB0032-SUB0036. This operon is absent in EF20 and isolate C9359 but is present in all other sequenced strains. Analysis of the 0140J genome identified five genes encoding putative bacteriocin proteins (SUB0502, SUB0505, SUB0506, SUB0509 and SUB0512) [6], of which SUB502-SUB505 are again absent in EF20 and also isolates B362 and 6780 both of which belong to the ST-86 complex. Whilst bacteriocin production does not define clinical and sub-clinical strains, the absence of almost all bacteriocins in the EF20 genome could put it at a competitive disadvantage with other environmental strains in the dairy cow environment and may reflect (but not explain) it's non-virulent status.

\section{Conclusions}

The comparison of multiple strains of closely related bacteria provides a valuable resource for the understanding of biological systems. The comparison of 12 newly sequenced strains together with the type 0140J strain of Streptococcus uberis allows a first comparison of bacteria isolated from clinical and non-clinical infections and the

\begin{tabular}{|c|c|c|}
\hline & $\begin{array}{ll}-79 & -75\end{array}$ & -10 \\
\hline $0140 \mathrm{~J}$ & TTTCTCGTAT & CTTEGTC \\
\hline Ab71 & TTTCTCGTATTAATP & TTGAAGTACCCTATCACATCATAATCATTGCGACCGATTCTTGGTCCATATTAAGGAGATCCGATGTATATCAAAC \\
\hline S6261 & TTTTCTCGTATTAATF & GAAAGTTGAAGTACCCTATCA CATCAT AATCATTGCGA CCGATTCTTGGTCCAT AT TAAGGA GATCCGA TGTA TA TCAAAC \\
\hline C5072 & TTTTCTCGTATTAATP & ATAGT AGAAAGTT GAAGTACCCTATCACA TCATAATCATTGCGACCGATTCTTGGTCCAT AT TAAGGAGACCCGA TGTATATCAAAC \\
\hline C6344 & TTTTCTCGTATTAATF & AAAGTT GAAGTACCCTATCA CA TCATAATCATTECGACCGATTCTT GGTCCATAT TAAGGA GACCCGATGTATATCAAAC \\
\hline C9359 & ITTTCTCGTATTAATZ & AAAGTTGAAGTACCCTATCA CATCATAATCATTECGACCGATTCTTEGTCCATATTAAGGAGATCCEATGTATATCAAAC \\
\hline 6780 & TTTCTCETATTCATF & ATAGTAGAAAGTTEA GTACCCTATCA CA TCATAATCATTECGACCGATTCTTETCCATATTAAGGA GATCCEATGTATA TCAAACA \\
\hline EF20 & TTTTCTCGTATTAATR & ATA GTAGAAAGTT GAA GTACCCTA TCA CA TCATAA TCA TTECGA CCGAT TCTTEGTCCAT AT TAAGGA GATCCEA TGTA TA TCAAA CA. \\
\hline B362 & TTTTCTCGTATTAATR & AAAGTTGAAGTACCCTATCACA TCAT AA TCA TTGCGA CCGAT TCTTGGTCCAT AT TAAGGA GA TCCGA TGTA TA TCAAA CAP \\
\hline $\operatorname{c53} 88$ & TTTTCTCGTATTAATF & AAGTT GAAGTACCCTATCA CA TCATAATCATT GCGACCGATTCTTGGTCCATAT TAAGGA GATCCGA TGTATA TCAAACAP \\
\hline $\mathrm{C} 8329$ & TTTTCTCGTATTA & AAGTT GAAGTACCCTATCA CATCATAATCATTGCGA CCGATTCTTGGTCCATAT TAAGGAGATCCGATGTATA TCAAACAP \\
\hline 6736 & ITTTCTCGTATICATZ & ATAGT AGAAAGTT GAAGT ACCCTATCA CATCATAATCATTGCGACCGATTCTTGGTCCATAT TAAGGA GATCCGA TGTATATCAAA CA \\
\hline B190 & & CCTATCACATCATAATCATTG \\
\hline
\end{tabular}

Figure 7 Variation in the vru upstream regions. Alignment of upstream region of vru gene across 13 strains was generated using Muscle [17]. Boxshade server (http://www.ch.embnet.org/software/BOX_form.html) was used to highlight the high degree of conservation within the aligned region. The position of the initiating methionine codon (ATG) is shown with an arrow. Deletion of TATAA was found in isolates EF20, 6736 and 6780 in position -75 to -79 of vru gene. Polymorphism of A to T found in region -79 along with the deletion of four bases TATA in regions -75 to -78 of isolates B362 and C8329. Deletion of four bases ATAA (-76 to -79) was found in 3 isolates Ab71, C5388 and C9359. 


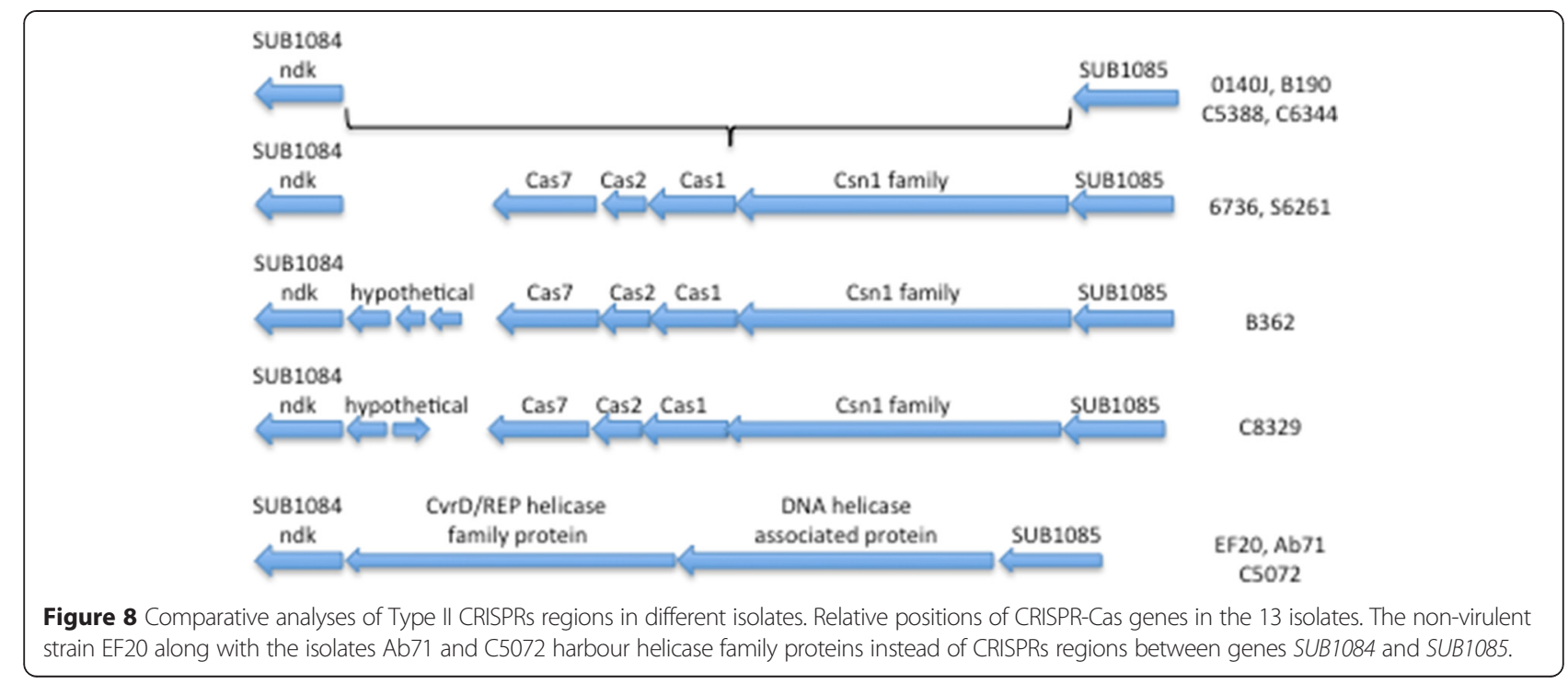

generation of a draft genome of EF20 strain together with the existing 0140J genome, allows for the first time comparison of two naturally occurring strains of S. uberis with defined virulence. The comparison of the strains did not suggest an obvious "smoking gun" gene either present or absent between the virulent or avirulent strains to suggest a previously unknown virulence factor. In addition the genome content did not differentiate between clinical and non-clinical strains. However, it is worth considering that the status as clinical or non-clinical refers to the state of the host animal from which the isolate was obtained, not to the causative agent. For example the proven non-virulent strain EF20 was isolated from a clinical case and hence is named as a clinical strain but this may have been due to other factors such as a co-infection with another bacterial species/strain and importantly, the genetics of the host. Thus, whilst the data here present a detailed comparison of $S$. uberis bacterial strains, to fully understand virulence and causation of disease, we must take a holistic approach encompassing bacteria, host and environment.

Table 3 Distribution of prophage regions among 13 isolates

\begin{tabular}{|c|c|c|c|c|c|c|c|}
\hline Strain & Region & Length & Status & \# CDS & Putative phage & GC \% & Location compared to $0140 \mathrm{~J}$ \\
\hline 0140J & 1 & & incomplete & 28 & Lactococcus_phage_blL311 & 33.6 & SUB1818-SUB1840 \\
\hline EF20 & None & & & & & & \\
\hline 6736 & 1 & $33.5 \mathrm{~Kb}$ & intact & 51 & PHAGE_Strept_5093_NC_012753 & 35.1 & SUB1470-SUB1471 \\
\hline 6780 & 1 & $35.3 \mathrm{~Kb}$ & questionable & 23 & PHAGE_Strept_PH10_NC_012756 & 40.6 & SUB1176-SUB1187 \\
\hline Ab71 & 1 & $12.9 \mathrm{~Kb}$ & incomplete & 15 & PHAGE_Lactoc_blL311_NC_002670 & 33.1 & SUB1818-SUB1840 \\
\hline B190 & 1 & $39.4 \mathrm{~Kb}$ & intact & 56 & PHAGE_Strept_pyogenes_315_1_NC_004584 & 34.9 & SUB0062-SUB0065 \\
\hline B362 & None & & & & & & \\
\hline \multirow[t]{2}{*}{ C5072 } & 1 & $47.7 \mathrm{~Kb}$ & questionable & 58 & PHAGE_Strept_pyogenes_315_2_NC_004585 & 39.7 & SUB1531-SUB1532 \\
\hline & 2 & $15.8 \mathrm{~Kb}$ & incomplete & 25 & PHAGE_Lactoc_blL311_NC_002670 & 32.8 & SUB1818-SUB1840 \\
\hline C5388 & 1 & $40.8 \mathrm{~Kb}$ & intact & 57 & PHAGE_Strept_P9_NC_009819 & 37.4 & SUB1748-SUB1452 \\
\hline \multirow[t]{2}{*}{ C6344 } & 1 & $46.6 \mathrm{~Kb}$ & questionable & 58 & PHAGE_Strept_pyogenes_315_2_NC_004585 & 38.5 & SUB1531-SUB1532 \\
\hline & 2 & $39.6 \mathrm{~Kb}$ & intact & 54 & PHAGE_Strept_SMP_NC_008721 & 36.8 & SUB1748-SUB1452 \\
\hline \multirow[t]{3}{*}{ C8329 } & 1 & $11.5 \mathrm{~Kb}$ & incomplete & 15 & PHAGE_Staphy_phi2958PVL_NC_011344 & 40.2 & SUB1183-SUB1190 \\
\hline & 2 & $45.7 \mathrm{~Kb}$ & intact & 56 & PHAGE_Strept_phi3396_NC_009018 & 36.5 & SUB1531-SUB1532 \\
\hline & 3 & $37.6 \mathrm{~Kb}$ & questionable & 38 & PHAGE_Lactoc_blL311_NC_002670 & 35.3 & SUB1818-SUB1840 \\
\hline C9359 & 1 & $40.8 \mathrm{~Kb}$ & intact & 57 & PHAGE_Strept_P9_NC_009819 & 37.4 & SUB1748-SUB1452 \\
\hline S6261 & 1 & $51.8 \mathrm{~Kb}$ & intact & 68 & PHAGE_Strept_TP_J34_NC_020197 & 36.4 & SUB1263-SUB1270 \\
\hline
\end{tabular}




\section{Database submission}

Sequence reads and assembled contigs are available at GenBank under accession JANW00000000, JATB0000 0000, JATD00000000, JATK00000000, JATE00000000, JATC00000000, JATI00000000, JATF00000000, JATA0 0000000, JATG00000000, JATJ00000000, JATH0000 0000 .

\section{Additional files}

\section{Additional file 1: Characteristics of the Streptococcus uberis isolates used in the study.}

Additional file 2: Genome annotation of 12 isolates using RAST server. Downloaded 22/06/14

\section{Additional file 3: Identification of orthologous gene clusters}

between strains. The pan-genome analysis pipeline (PGAP version 1.02) [11] was used for identification of orthologous genes between the twelve sequenced genomes and the reference genome 0140J using the Gene Family (GF) method (50\% coverage and with an e-value cut-off 1e-10).

Additional file 4: Metabolic subsystem comparison between 0140J and EF20 predicted by RAST server. Downloaded 22/06/14.

\section{Competing interests}

The authors declare that they have no competing interests.

\section{Authors' contributions}

$\mathrm{MH}$ and RDE assembled the genomes and conducted the comparative genomics annotation and experiments. RW generated sequence libraries and reads. SE provided genomic DNA for sequencing along with PNW who also conducted preliminary genome analysis of strain EF20. TJC, JAL and SAE conducted the EF20 and 0140J challenge experiments. MH, SE, JAL and RDE wrote the manuscript. JAL and RDE conceived and oversaw the study. All authors read and approved the manuscript.

\section{Acknowledgements}

We acknowledge the financial support of BBSRC (grant numbers E0181141 $(\mathrm{PI}=\mathrm{TC})$ and $\mathrm{E} 0181732(\mathrm{PI}=\mathrm{L}, \mathrm{COI}=\mathrm{PW}$, supported researcher $=\mathrm{SE}), \mathrm{DFERA}$ (grant number OD1717 PI $=J \mathrm{~L}$ ) and the University of Nottingham. MH was supported by a University of Nottingham Vice Chancellors international scholarship award. We gratefully acknowledge Adam Blanchard School of Veterinary Medicine and Science and Tom Giles and Andrew Warry Advanced Data Analysis Centre University of Nottingham for helpful discussions.

\section{Author details}

${ }^{1}$ School of Veterinary Medicine and Science, University of Nottingham, Leicestershire, LE12 5RD Sutton Bonington, UK. ${ }^{2}$ Sir William Dunn School of Pathology, The University of Oxford, Oxford, UK. ${ }^{3}$ DeepSeq, School of Life Sciences, University of Nottingham, Queen's Medical Centre, Nottingham, UK. ${ }^{4}$ Advanced Data Analysis Centre, University of Nottingham, Nottingham, UK.

Received: 23 October 2014 Accepted: 8 April 2015

Published online: 23 April 2015

\section{References}

1. Bramley AJ, Dodd FH. Reviews of the progress of dairy science: mastitis control-progress and prospects. J Dairy Res. 1984;51(3):481-512.

2. Bradley AJ, Leach KA, Breen JE, Green LE, Green MJ. Survey of the incidence and aetiology of mastitis on dairy farms in England and Wales. Vet Rec. 2007;160(8):253-7.

3. Bradley AJ, Barkema H, Biggs A, Green MJ, Lam T. Control of mastitis and enhancement of milk quality. In: Green MJ, editor. Dairy Herd Health. Oxfordshire, UK: CABI; 2012

4. Bradley $A B H$, Biggs A, Green M, Lam T. Control of mastitis and enhancement of milk quality. In: Green M, editor. Dairy herd health. UK: CPI Group (UK) Ltd; 2012
5. Leigh JA. Streptococcus uberis: a permanent barrier to the control of bovine mastitis? Vet J. 1999;157(3):225-38.

6. Ward PN, Holden MT, Leigh JA, Lennard N, Bignell A, Barron A, et al. Evidence for niche adaptation in the genome of the bovine pathogen Streptococcus uberis. BMC Genomics. 2009;10:54.

7. Leigh JA, Egan SA, Ward PN, Field TR, Coffey TJ. Sortase anchored proteins of Streptococcus uberis play major roles in the pathogenesis of bovine mastitis in dairy cattle. Vet Res. 2010;41(5):63.

8. Zerbino DR. Using the Velvet de novo assembler for short-read sequencing technologies. Curr Protoc Bioinformatics. 2010;11:11-5.

9. Galardini M, Biondi EG, Bazzicalupo M, Mengoni A. CONTIGuator: a bacterial genomes finishing tool for structural insights on draft genomes. Source Code Biol Med. 2011;6:11.

10. Aziz RK, Bartels D, Best AA, DeJongh M, Disz T, Edwards RA, et al. The RAST Server: rapid annotations using subsystems technology. BMC Genomics. 2008:9:75.

11. Zhao Y, Wu J, Yang J, Sun S, Xiao J, Yu J. PGAP: Pan-Genomes Analysis Pipeline. Bioinformatics. 2011;28(3):416-8.

12. Edgar RC. PILER-CR: fast and accurate identification of CRISPR repeats. BMC Bioinformatics. 2007;8:18.

13. Grissa I, Vergnaud G, Pourcel C. CRISPRFinder: a web tool to identify clustered regularly interspaced short palindromic repeats. Nucleic Acids Res. 2007;35:W52-7.

14. Grissa I, Vergnaud G, Pourcel C. The CRISPRdb database and tools to display CRISPRs and to generate dictionaries of spacers and repeats. BMC Bioinformatics. 2007;8:172.

15. Grissa I, Vergnaud G, Pourcel C. CRISPRcompar: a website to compare clustered regularly interspaced short palindromic repeats. Nucleic Acids Res. 2008:36:W145-8.

16. Zhou Y, Liang Y, Lynch KH, Dennis JJ, Wishart DS. PHAST: a fast phage search tool. Nucleic Acids Res. 2011;39:W347-52

17. Edgar RC. MUSCLE: multiple sequence alignment with high accuracy and high throughput. Nucleic Acids Res. 2004;32(5):1792-7.

18. Leekitcharoenphon P, Kaas RS, Thomsen MC, Friis C, Rasmussen S, Aarestrup FM. snpTree-a web-server to identify and construct SNP trees from whole genome sequence data. BMC Genomics. 2012;13(7):S6.

19. Guindon S, Gascuel O. A simple, fast, and accurate algorithm to estimate large phylogenies by maximum likelihood. Syst Biol. 2003;52(5):696-704.

20. Francisco AP, Vaz C, Monteiro PT, Melo-Cristino J, Ramirez M, Carrico JA. PHYLOViZ: phylogenetic inference and data visualization for sequence based typing methods. BMC Bioinformatics. 2012;13:87.

21. Field TR, Ward PN, Pedersen LH, Leigh JA. The hyaluronic acid capsule of Streptococcus uberis is not required for the development of infection and clinical mastitis. Infect Immun. 2003;71(1):132-9.

22. Smith AJ, Ward PN, Field TR, Jones CL, Lincoln RA, Leigh JA. MtuA, a lipoprotein receptor antigen from Streptococcus uberis, is responsible for acquisition of manganese during growth in milk and is essential for infection of the lactating bovine mammary gland. Infect Immun. 2003;71(9):4842-9.

23. Hill AW. Pathogenicity of two strains of Streptococcus uberis infused into lactating and non-lactating bovine mammary glands. Res Vet Sci. 1988:45(3):400-4.

24. Tettelin H, Masignani V, Cieslewicz MJ, Donati C, Medini D, Ward NL, et al. Genome analysis of multiple pathogenic isolates of Streptococcus agalactiae: Implications for the microbial "pan-genome". Proc Natl Acad Sci. 2005:102(39):13950-5.

25. Bruen T, Phillipe $H$, Bryant D. A quick and robust statistical test to detect the presence of recombination. Genetics. 2006;17:2665-81.

26. Martin PR, Hoiby EA. Streptococcal serogroup A epidemic in Norway 1987-1988. Scand J Infect Dis. 1990;22(4):421-9.

27. Cho KH, Caparon MG. Patterns of virulence gene expression differ between biofilm and tissue communities of Streptococcus pyogenes. Mol Microbiol. 2005;57(6):1545-56.

28. Ward PN, Field TR, Ditcham WG, Maguin E, Leigh JA. Identification and disruption of two discrete loci encoding hyaluronic acid capsule biosynthesis genes has A, hasB, and hasC in Streptococcus uberis. Infect Immun. 2001;69(1):392-9.

29. Ashbaugh CD, Alberti S, Wessels MR. Molecular analysis of the capsule gene region of group $A$ Streptococcus: the has $A B$ genes are sufficient for capsule expression. J Bacteriol. 1998;180(18):4955-9. 
30. Egan SA, Ward PN, Watson M, Field TR, Leigh JA. Vru (Sub0144) controls expression of proven and putative virulence determinants and alters the ability of Streptococcus uberis to cause disease in dairy cattle. Microbiology. 2012;158(Pt 6):1581-92

31. Flores AR, Olsen RJ, Wunsche A, Kumaraswami M, Shelburne 3rd SA, Carroll RK, et al. Natural variation in the promoter of the gene encoding the Mga regulator alters host-pathogen interactions in group a Streptococcus carrier strains. Infect Immun. 2013;81(11):4128-38.

32. Deveau H, Garneau JE, Moineau S. CRISPR/Cas system and its role in phage-bacteria interactions. Annu Rev Microbiol. 2010;64:475-93.

33. Horvath P, Barrangou R. CRISPR/Cas, the immune system of bacteria and archaea. Science. 2010;327(5962):167-70

34. Karginov FV, Hannon GJ. The CRISPR system: small RNA-guided defense in bacteria and archaea. Mol Cell. 2010;37(1):7-19.

35. Koonin EV, Makarova KS. CRISPR-Cas: an adaptive immunity system in prokaryotes. F1000 Biol Rep. 2009;1:95.

36. Sorek R, Kunin V, Hugenholtz P. CRISPR-a widespread system that provides acquired resistance against phages in bacteria and archaea. Nat Rev Microbiol. 2008;6(3):181-6.

37. van der Oost J, Jore MM, Westra ER, Lundgren M, Brouns SJ. CRISPR-based adaptive and heritable immunity in prokaryotes. Trends Biochem Sci. 2009;34(8):401-7.

38. Makarova KS, Haft DH, Barrangou R, Brouns SJ, Charpentier E, Horvath P, et al. Evolution and classification of the CRISPR-Cas systems. Nat Rev Microbiol. 2011;9(6):467-77.

39. Brussow H, Canchaya C, Hardt WD. Phages and the evolution of bacteria pathogens: from genomic rearrangements to lysogenic conversion. Microbiol Mol Biol Rev. 2004:68(3):560-602.

40. Alikhan NF, Petty NK, Ben Zakour NL, Beatson SA. BLAST Ring Image Generator (BRIG): simple prokaryote genome comparisons. BMC Genomics. 2011;12:402.

\section{Submit your next manuscript to BioMed Central and take full advantage of:}

- Convenient online submission

- Thorough peer review

- No space constraints or color figure charges

- Immediate publication on acceptance

- Inclusion in PubMed, CAS, Scopus and Google Scholar

- Research which is freely available for redistribution 